\title{
Work Performance of Railway Station Plan Terminal Over CTC System
}

\author{
Ziyuan Liu ${ }^{1, a}$ \\ 1Department of Automatic Traffic Control, Signal \& Communication Research Institute, China \\ Academy of Railway Science, Daliushu Road 2, Haidian District, Beijing, China \\ a38356253@qq.com
}

\begin{abstract}
Keywords: Centralized Train Control (CTC), Station Plan Terminal(SPT), flexible plan, efficient scheduling, track modulation.
\end{abstract}

Abstract. Traffic Dispatching and Command System works as the train dispatching center to make the operational decision on almost every train. The new generation dispatching system called Centralized Train Control (CTC) System works as the train dispatching command system of the China's high-speed rail. The center dispatcher terminal of CTC is the only plan decider to make the final decision on each plan and send them to the stations in its section. In order to make the plan more flexible, this paper put forward a new terminal Station Plan Terminal (SPT) embedded in the existing CTC system to server as the final plan decider. SPT is the terminal used by station dispatcher to receive phase plan from the center and modulate them according to the station situation. After introduction of the new system, we did some simulation and analysis on the function of SPT working in the integrated CTC system. The methodology in our simulation is using SPT to modulate plans according to the station situation, and sent all the plans including the modulated ones to the autonomous machine. The whole work flow of the simulation traverse all the information flow control and logic of SPT. Through the simulation results, we can get that plans refined by SPT and were sent to the autonomous machine took more accurate on route execution, so that the work flow efficiency of the whole dispatching system can be enhanced to some extent.

\section{Introduction}

Recent fast development in computer technology enable automatic control system accomplished and used in traffic control of the railway. Signal and Communication system not only keep the train safe during the high-speed running but also support the passing capability through the railway section in a high degree.

Beyond the primary purpose of operation safety, researchers and workers pursue the maximum passing ability of the station and the section. The plan made by computer technology are used in the China Train Dispatching and Command System and the enhanced system which is called Centralized Train Control system (CTC) used in the dedicated passenger lines and high-speed lines take advantage of autonomous control technology. The final decision of almost all the plan is made and by the center dispatcher who use the plan management terminal of CTC. The plan formed in the terminal sent to the station autonomous machine which can solve the information and transform the plan into the autonomous route list. The route can be triggered by the station autonomous machine at the trigger point.

After serval years CTC system operation on the existing train line, center dispatcher dose all the determination on the plan have its obvious advantages. One of them is taking the whole section into the consideration, the utilization of the railway section capacity can be allocated in a high level. However, the real-time condition of each station yard cannot be considered by the center dispatcher. All the stations with a large quantity of work have strong demand of decentralizing the plan modulation to each station dispatcher themselves. If so a lot of extra human communication work can also be eliminated which will reduce the work load of center dispatchers and even more the fault can be also reduced.

The aim of this paper is to propose a more efficient system and work flow to support a more flexible train dispatching and command [1]. In the existing railway dispatching operation, the center dispatcher is the final plan decider. What the station dispatcher do to affect the plan is using digital 
dedicated line phone to negotiate with the center dispatcher. The process of negotiation adds some extra work to both side and phase plans cannot be modulated according to the change of the station yard in time.

This paper is organized as follows. Section II will discuss the new architecture and the information architecture of the system after the new station plan terminal (SPT) joining in the current CTC system. The work flow and information flow of the new terminal will be introduced in the Section III and in Section IV we will present the test result of the terminal software, this paper will be concluded in Section V.

\section{New CTC System Architecture}

In this section, our new integrated CTC system are founded and illustrated in details.

System Architecture Overview. The relative node in the new CTC system topology is shown in Fig.1. The Station Plan Terminal connected with the station communicator to embed into the system network. The SPT program is embed into the system network through connect to the station communicator which is used as the gateway to support network access. The Plan from the center plan terminal (CPT) is first transferred by the center communication center to the station communicator and then to SPT.

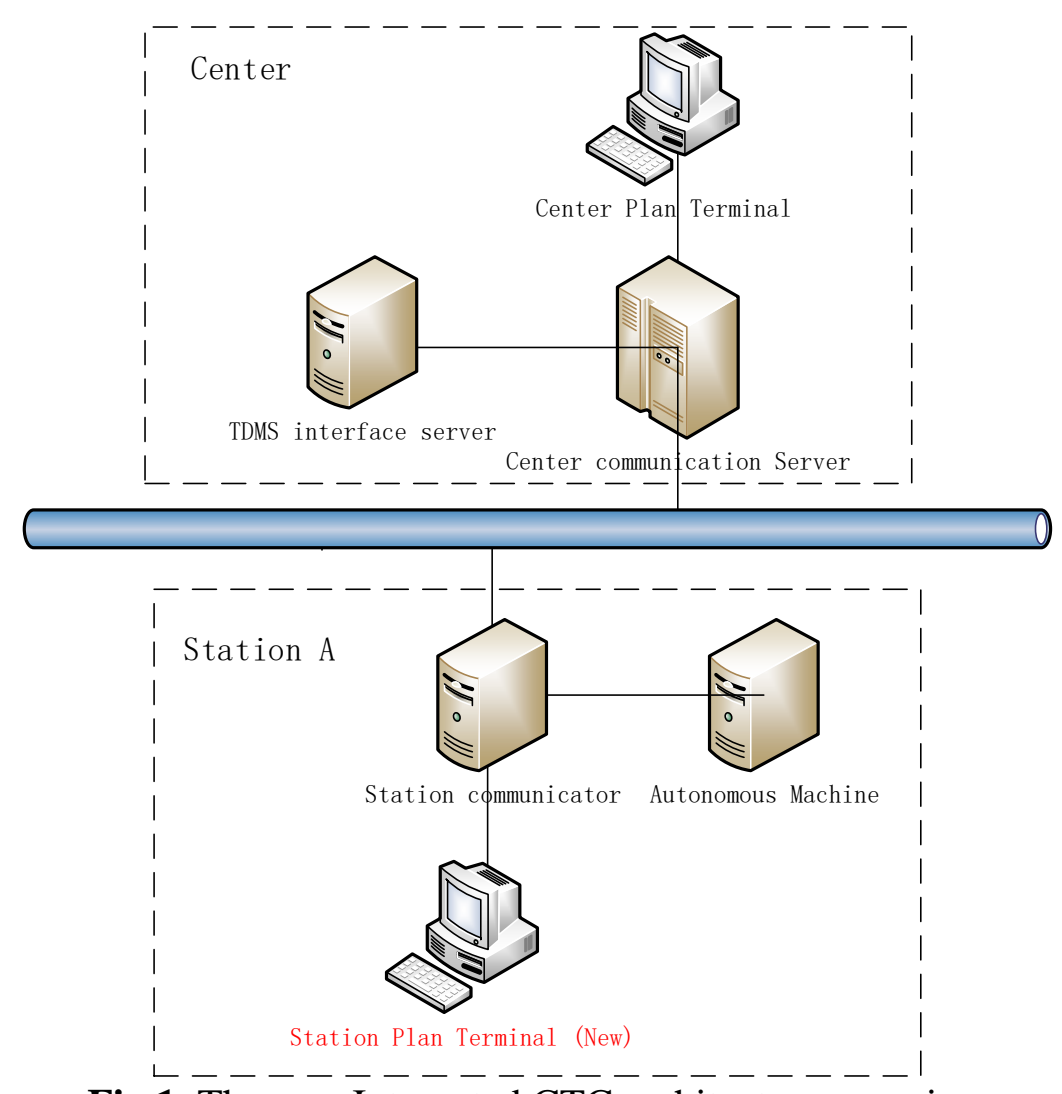

Fig.1. The new Integrated $\bar{C} \overline{\text { TC }}$ architecture overview

Information Architecture Overview. Fig.2 shows all the information flow between SPT and CTC system. Among of them, station communicator like a hub to transfer all the information sent from or received by SPT. SPT needs Phase Plan originated from CPT to be as the base. It needs sufficient and necessary check on the safety of plan modulation, daily plan information, display information of the station and route status of the corresponding plan are all the legal ground of the plan track modulation. 


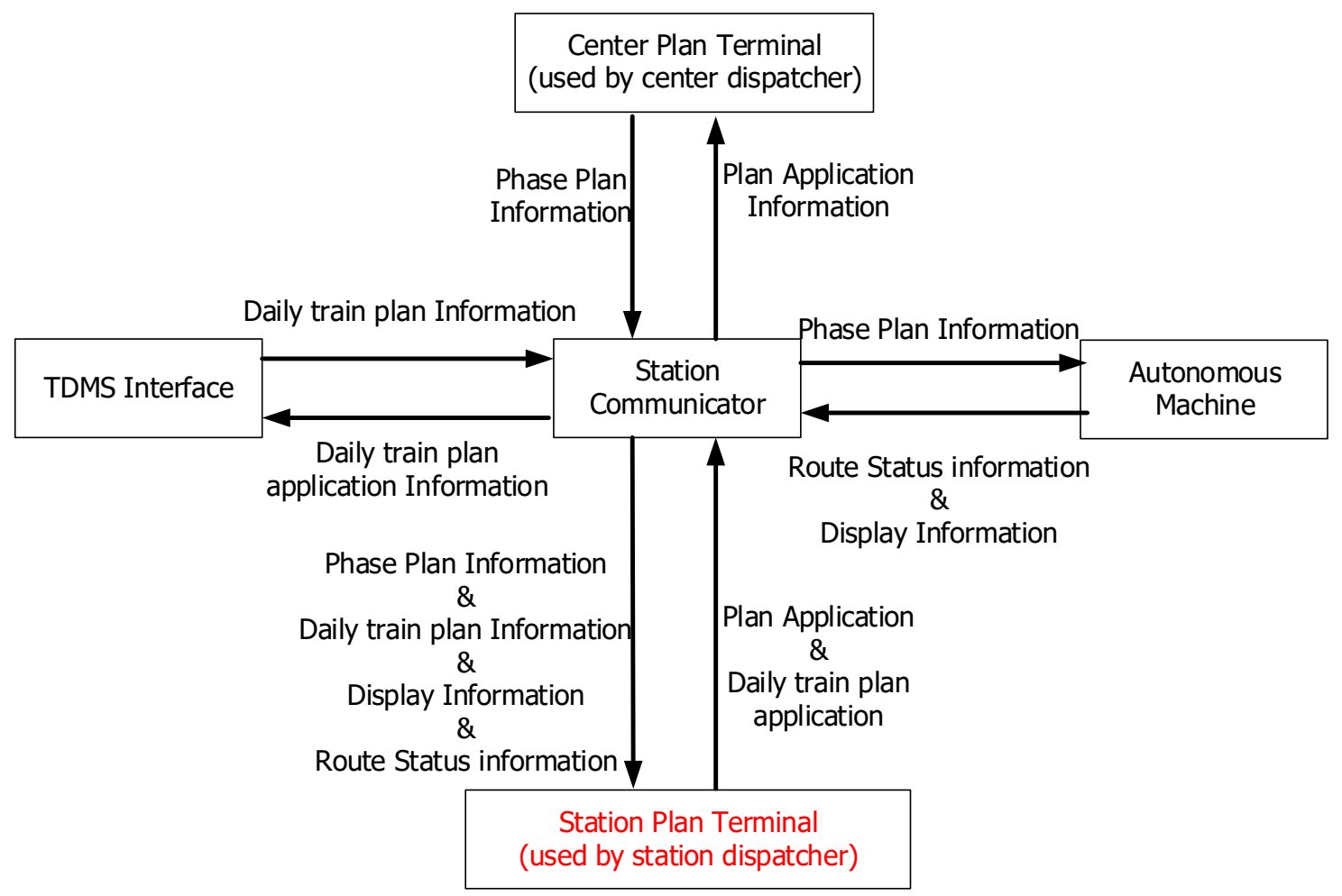

Fig.2. STP Information architecture overview

\begin{tabular}{|l|l|}
\hline $\begin{array}{l}\text { Phase Plan Application } \\
\text { Information }\end{array}$ & $\begin{array}{l}\text { SPT send the information dedicated to the CPT through the } \\
\text { station communicator }\end{array}$ \\
\hline $\begin{array}{l}\text { Phase Plan Information } \\
\text { (from CPT) }\end{array}$ & $\begin{array}{l}\text { the CPT send phase plan broadcast, and the STP can receive the } \\
\text { information as one of the terminal in the CTC system }\end{array}$ \\
\hline Display information & $\begin{array}{l}\text { autonomous machine send the real-time display information } \\
\text { broadcast and the SPT can receive the information as one of the } \\
\text { terminal in the CTC system }\end{array}$ \\
\hline $\begin{array}{l}\text { Daily Plan Application } \\
\text { information }\end{array}$ & $\begin{array}{l}\text { Originated from STP to apply daily plan to TDMS interface fixed } \\
\text { time. }\end{array}$ \\
\hline Daily Plan information & $\begin{array}{l}\text { TDMS interface server send the fixed daily plan at a regular time } \\
\text { dedicated to SPT }\end{array}$ \\
\hline Route Status information & $\begin{array}{l}\text { autonomous machine send the real-time route status information } \\
\text { broadcast and the SPT can receive the information as one of the } \\
\text { terminal in the CTC system }\end{array}$ \\
\hline $\begin{array}{l}\text { Phase Plan Information } \\
\text { (from SPT) }\end{array}$ & $\begin{array}{l}\text { The phase plan is sent broadcast by SPT, which can be received } \\
\text { by the autonomous machine. }\end{array}$ \\
\hline
\end{tabular}

Table.1. Information flow of the new integrated CTC system

The listed information in Fig.1 and Table.1 of the new integrated CTC system forms Closed loop to support the whole work flow of the new integrated system.

\section{Simulation Modules}

SPT is connected to the station communicator to be integrated into the CTC system, which the logic of the software should also conform to the logic of the existing system to work.

Integrated new CTC system module. The module we used for our simulation is based on the new CTC architecture showed in Fig.1, which is made up of the center devices: database server, CPT, center communication server, TDMS and the station devices: station communicator, autonomous machine, SPT. 
Simulation Methodology. In the simulation, we did test according to the work flow related to SPT in Fig.3. ellipse represent the related terminal of the integrated CTC system and the contents of the rectangle under each ellipse are the work details of each terminal.

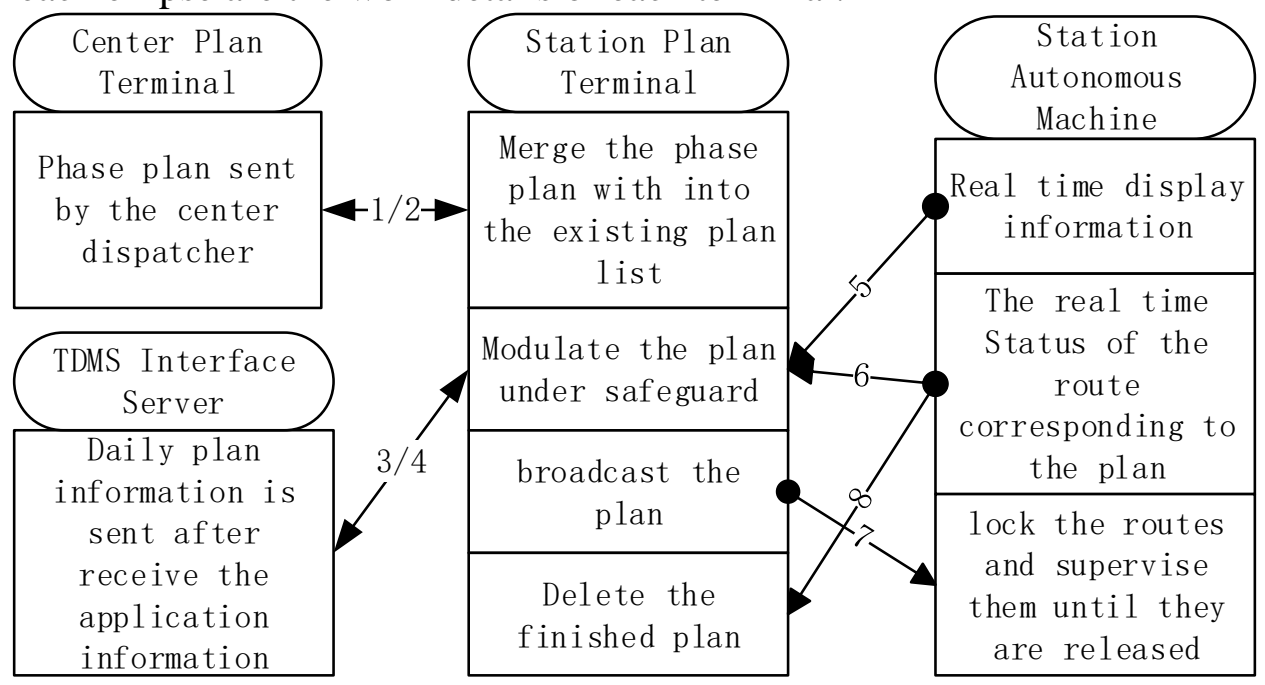

Fig.3. STP workflow over the whole CTC system overview

Process 1/2: the information transmission between the CPT and SPT is the phase plan dealing process. At the start of the terminal program, the Application for the phase plan of the station is sent dedicated to CPT which administrate the local station. CPT will inform the dispatcher with the tip and the phase plan will be sent manually. SPT will solve the phase plan information and display them on the grid of the software. The application is only triggered at the time of the start of the program, and in the whole running process, the plan list in the terminal will be kept up to date using the existing logic which the dispatcher will manually send the phase plan after any change.

Process 3/4: TDMS interface supports the daily plans which include all the information of each plan used as the judging condition to modulate the plan on track item.

Process 5: The real-time display information of the whole station sent by the autonomous machine of the same station support the judging conditions of plan modulation by SPT.

Process 6: The route status of each plan sent by the autonomous machine is used by the terminal as the judging condition if it can be any change of the plan. If the route is already triggered, the corresponding plan should not be changed anymore.

Process 7: After station dispatcher do his operation on the phase plan, he will send the final plans broadcast and one of the plan destination is autonomous machine which will form route table corresponding to each plan.

Process 8: If the route status information is that the route is released after the train is arriving the terminal or passing through the station, SPT will delete the corresponding plan from the software memory and the interface table.

Summary of above, the simulation module and the simulation methodology is sufficient for us to do the simulation. We will do simulation and get results of the system function to evaluate the SPT performance over the new integrated CTC system in the next section.

Simulation results. The main object of the simulation is mainly to verify that all the phase plans received from the center can modulated under the train safety guard of software. And after that the plan can be accurately executed by the autonomous machine.

At the very beginning of the simulation, we established the new CTC system using the architecture topology shown in Fig.1. the simulation module was the existing CTC system which including CPT, Center Communicator Server, TDMS Interface Server, Station communicator and Autonomous Machine. Then we launched SPT Program on the station terminal. The icon of the network state on the interface of SPT program shows that it successfully connects to the station communicator through TCP/IP network. After that, the new CTC system is integrated completely and we used this system to do the further simulation. 
First of all, we got a pop-up message box that "xxx station apply the phase plan" on the CPT interface, which proves that the 'Plan Application information' is transmitted and solved correctly. Follow the work flow, then we used CPT to send the phase plan manually as the center dispatcher. We checked the SPT interface and got that the plans are listed row by row user interface in the sequence of the arrival time. Each row of the grid fill the items of each plan in order, "Back station", "Train Number", "Arrive Time", "Departure Time", "Track", "Front Station". It proves that the phase plan information is solved correctly by the SPT.

Second, we do modulation on the plans showed on the SPT interface. We chose one of the plans and click the 'track' item, a combo box showed all the tracks of the station to be chosen, we randomly choose a track different from the original one. Then we got a message box popup on the screen to shows that "the target track is different from the one of Daily Train Plan". This is the result that we have already complete the Daily Train Plan information close-looped transmission and solve the information correctly.

In order to verify the safety control, which is the basement of our SPT software, we traverse all station status and do modulation on 'track' item to test the safety assurance made by the software to the plans.

Track modulation Alarms: to the station dispatcher of our software are that the operation on the plan cannot be continued for the reason of violating safety rules. All the alarms triggered by our simulation are listed in Table.2.

1.Whether the new track and the port can be formed the effective route.

2.Passenger train forbid to pass through the under 12-sized switch.

3.Freight train forbid to pass through the under 9-sized switch

4.Over-limit vehicles are forbidden to access into the normal track.

5.An over-limit vehicle is forbidden to access into the track on which neighbor track another Over-limit vehicle is already occupied;

6. The electric-tract train forbid to access into the track without catenary (overhead contact line).

7.The passenger train which has boarding work in the station should board(dock) at the track with platform.

8.The main line of the station should not accept the train to board.

Table.2. Track modulation alarms

Track Modulation Cautions: under some conditions, the plan can be modulated to the target track after the confirmation by station dispatcher. All the cautions of our software are listed in Table.3.

1.The electric-tract train accessing into the track with temporary electric power cut should be ensured;

2.train accessing into the temporarily blocked track should be ensured;

3.The Electric multiple unit trains (EMU trains) arranged to board at the track with high platform violate regular rule.

4.Freight train arranged to board at the track with high platform violate the regular rule.

5.Passenger train passing through the side line without boarding violate the regular rule.

6.Freight train passing through the side line without boarding violate the regular rule.

Table.3. Track modulation cautions

All the alarms and cautions listed above can prove that the safety assurance provided by the software is necessary and comprehensive. After modulated by SPT, phase plans were the final edition that could not be changed anymore by the CPT. This is also verified through our simulation. The autonomous machine received phase plans from the SPT and served them as the final edition which were used to do the autonomous route locking to support the train running.

\section{Conclusions}

In this paper, we design and complete SPT software with the mainly purpose of improving the efficiency and reasonability of the existing CTC system. By the method of our simulation we test SPT software function and analyzed the performance of SPT working in the new integrated CTC system. 
From our simulation result, we draw the conclusion that firstly SPT can be embedded into the existing CTC system to form a new integrated CTC system with close loop information. Secondly it can be used to modulate track of each plan properly under the safeguard function of SPT, so that plans can be solved flexibly and accurately without delay. In the new CTC system, Plans sent by SPT are the final train traffic diagram on which automatic route control is based. Further benefits are as follows: amount of redundant work between the center and the station can be remarkably decreased, the delay of call waiting to negotiate the plans are remarkable reduced. And above all, the proposal of SPT in this paper facilitates better use of each station and every sector handling capacity by precisely adjusting plans.

\section{References}

[1] Ludmila Janosikova; Antonin Kavicka; Michael Bazant: Optimal operation scheduling and platform track assignment in a passenger railway station, Proceedings of the Institution of Mechanical Engineers, Part F. Journal of rail and rapid transit, Vol. 228(2014), p. 271-284. 